\title{
ARIES: Enabling Visual Exploration and Organization of Art Image Collections
}

\author{
Lhaylla Crissaff, Louisa Ruby, Samantha Deutch, Luke DuBois, Jean-Daniel Fekete, Senior Member, \\ IEEE, Juliana Freire, Member, IEEE, Cláudio T. Silva, Fellow, IEEE
}

\begin{abstract}
Art historians have traditionally used physical light boxes to prepare exhibits or curate collections. On a light box, they can place slides or printed images, move the images around at will, group them as desired, and visually compare them. The transition to digital images has rendered this workflow obsolete. Now, art historians lack well-designed, unified interactive software tools that effectively support the operations they perform with physical light boxes. To address this problem, we designed ARIES (ARt Image Exploration Space), an interactive image manipulation system that enables the exploration and organization of fine digital art. The system allows images to be compared in multiple ways, offering dynamic overlays analogous to a physical light box, and supporting advanced image comparisons and feature-matching functions, available through computational image processing. We demonstrate the effectiveness of our system to support art historians' tasks through real use cases.
\end{abstract}

Index Terms-User Experience, User Interaction, Art History, Image Collections, Lightbox

\section{INTRODUCTION}

The field of Art History expanded rapidly with the advent of photography in the 19th century. For the first time, art historians had permanent access to images of works of art which they could use as reference tools for their writing and thinking on art. With the release in the past fifteen years of millions of images online, the field is once again experiencing a major transformation. Although a large number of works of art or even existing photographs of works of art in the world have yet to be digitized, having digital images has revolutionized the way art historians function. Rather than having to rely on analog photographs of varying quality, available only as part of either a personal slide or image library, art historians now have instant online access to a large number of high quality images.

Art historians study artistic artifacts: paintings, drawings, sculptures and architectures for various purposes such as defining their meaning, style, value and historical context in which they were created. For an art historian, images are the foundation of all research; they are the examples, use cases, test cases, subjects, objects, and purpose behind all of their work. Before the digital age, art historians used reproducible physical media such as prints (etchings or lithographs), photographs, or slides as memory aids for works of art they had seen and wanted to discuss. These physical objects can be moved around on tables or light boxes (Fig. 11) and organized into different piles and groupings for further examination.

Today, art historians mostly work with digital images, but their needs have not been adequately met: there are no software tools that support the organization, editing, use, and flow of images needed to support their work methodology. As a result, working with digital images is timeconsuming, tedious, and requires ad-hoc configurations of software tools to perform the basic tasks required in their field.
To address this problem, we have designed ARIES (ARt Image Exploration Space), a new interactive system that better supports art historians in their daily workflow. ARIES was developed as part of a collaboration with professional art historians from the Frick Collection, one of the richest privately-held art collections in the United States and a prestigious art museum in New York City. The system simplifies the exploration, analysis, and organization of digital image collections by allowing experts to easily manipulate digital images like they used to manipulate printed images, and combining this functionality with advanced tools for comparing images and matching features.
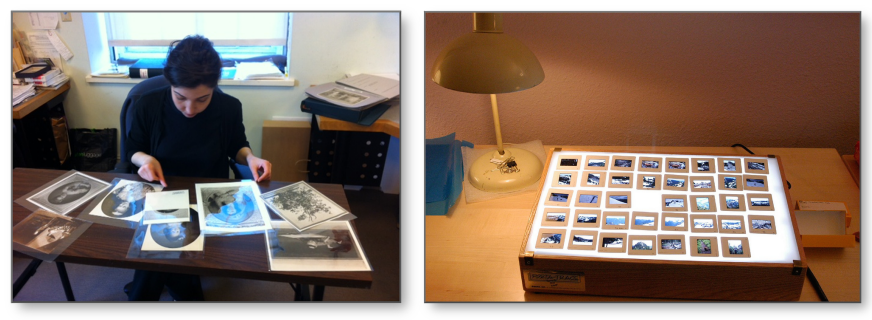

Fig. 1. In the physical world, art historians work with photographs and slides that can be moved around on tables (left) or light boxes (right).

Our main contributions can be summarized as follows:

- We present the tasks and requirements that influenced the design of ARIES and discuss how we addressed them.

- We describe the ARIES integrated environment and its components, including the virtual lightbox and a set of tools that support grouping, organization, annotation and comparison of images.

- We report on the use of ARIES by art historians and discuss a set of use cases that demonstrate the effectiveness of the system. 


\section{BACKGROUND}

Tools have been designed to support the creation, sharing and integration of archives of digital objects. Fuchs et al.[1] and Smith et al. [2] presented VLMA, a peer-to-peer framework that facilitates viewing, collecting, and reusing distributed visual archives and associated metadata. By configuring VLMA on a server, a user can both share her files and obtain access to files shared by other VLMA users. Within a server, once the files are imported from other collections, it is possible to perform simple tasks such as arranging, scaling and adding annotations. Google's Cultural Institute [3] is a web-based collection exploration environment that allows users to create their own collections by importing fragments from other collections stored in Google's database. These include art collections from museums, galleries, and works of individual artists (e.g., street graffiti artists). Collections of images can be browsed, individual items can be examined in high resolution, shared, and it is possible to view two images side by side. Saklofske [4] created an exploratory tool to visualize artwork collections. In this tool, each artwork is a page in the archive, and each page is represented as a node in the visualization. Nodes can be repositioned, grouped, connected (by drawing a line between two nodes), and annotated. Like these approaches, ARIES helps users organize image collections. However, ARIES also provides operations that support analysis, exploration, and comparison of images as well as the ability to arrange multiple images freely on a screen that can be shared between users.

Ciocca et al. [5] proposed a system for browsing museum collections that employs with a multi-touch table as a user interface. Images from their database can be imported into the application through physical object interactions, and then be manipulated by applying rotation, scaling and translation using finger gestures on the touch interface. Multitouch tables systems have also been designed by Hinrichs et al. [6], Hornecker [7] and Davis et al. [8] to engage museum visitors in the exploration of museum exhibitions. In all cases, tabletops display information about artworks and visitors can select what to see, such as textual information, pictures and videos. Geller's survey [9] presents many successful tabletop systems used by museums. While these systems were designed for museum visitors, ARIES and its features were designed to support research in art history.

An important task required by art historians and not supported by any of these tools is the ability to compare images. In the Computer Graphics field, automatic techniques have been proposed for image comparison [10], [11]. In these works, keypoint detection and feature vector extraction enable the identification of matches between comparable images. Images that art historians compare usually show differences of degree, not kind, a nuance lost by simple automatic image comparison algorithms, which did not behave in way that would add to any of the tasks we were aiming to address. Instead of applying automated techniques that are not useful for art historians, we opted to approach image comparison in a user-driven fashion: we provide tools that enable users to focus on (important) details present in the artworks, and guide art historians as they classify the images.

In the domain of cartography, tools have been created to compare images, either to study evolution over time or to register multiple images. Lobo et al. [12] described and evaluated the effectiveness of multiple interactive techniques to compare two maps, that are images with a specific semantic. Their main task consisted of spotting changes between two images. Elias et al. [13] also proposed an interface for browsing and correlating large numbers of maps. Art historians also need to correlate images but, just like comparing images, these are only two of the many tasks they routinely perform while working on their images.

Currently, there are no tools designed specifically for art historians, working on their own, offline, and with images from their own collections. As a result, art historians are forced to use multiple tools to accomplish what the (physical) lightbox offered. Tools such as Pinterest and applications such as Adobe Photoshop, Adobe Bridge, Adobe Lightroom and Microsoft PowerPoint are commonly used but support only a subset of the functionality they need. Having to use multiple tools that are not properly integrated greatly hampers the experts' ability to analyze their data. ARIES focuses on the specific tasks performed by art historians and provides an integrated environment designed to support art historical research.

\section{Requirements}

Art historians usually work with images of paintings, drawings and sculptures taken not only from their own databases, but from sanctioned databases (so-called "special collections"), as well as the web. These images are organized into groups using criteria established for a given project, such as subject matter, artist, style, date, or location. Metadata is used to describe the work of art, such as title, date, medium, dimensions and provenance (history of locations the artwork has been and events it was part of). Images are visually compared to check for similarities and differences, duplicates, degradation or restorations, forged artworks, to see close ups of details, etc. Their findings are shared with colleagues from museums, galleries, schools and research centers around the world.

When we started this project, our goal was to create an environment to help art historians explore and organize art images in a virtual space. We wanted to bring the flexibility and freedom they enjoyed while manipulating images in light boxes to a digital environment.

The initial needs assessment for ARIES was done in collaboration with three professional art historians from the Frick Collection. We carried out extensive interviews to understand their workflow, how they handled art images, and what questions they need to answer in their research. Two of the art historians, co-authors of this paper, also tested the system, provided feedback during the development, and used it in real projects which we describe in Section 6.

After identifying the main tasks, an initial list of desired functionality was made based on art historians' experience in using other computational tools as Adobe PowerPoint, Adobe Bridge, and Pinterest. Initial mock-ups of a possible digital lightbox interface were developed by graduate students studying user experience and presented to the staff from the museum. Although each mock-up had some of the established required functionality, we had to combine 
many versions of the mock-ups to create an interface that supported multiple image manipulation functions. This iterative design phase was crucial for developing an interface focused on art historians' needs and still maintaining its simplicity.

The interviews phase allowed us to derive two sets of requirements. The basic requirements guided the design of the workspace to manipulate digital images. The workspace should allow the user to bring several images from multiple sources into a single environment and to work on them at will; work with the metadata associated with the images; add annotations to images; group and arrange images; save work-in-progress for later use; and share work in progress with collaborators. These features, despite being simple and already supported by other tools, enable ARIES to provide a unified framework that supports all the tasks in the the art historians' workflow.

The second set, the specific requirements, determined the functionalities needed to explore and compare images. The system should assist experts to detect, interpret and understand works of art and their relationships. In particular, it should support the ability to: superimpose images, allowing for a quick and easy determination of physical changes to works of art that may have taken place over time due to damage or conservation; the detection of forgeries and copies; and the instantaneous comparison of the differences between draft drawings and finished works of art. The system should also support complex visual comparisons of similar compositions by being able to quickly selecting analogous portions of multiple works of art and placing them side by side. This allows art historians to analyze an artist's myriad portrayals of a specific scene be it a painting, print or drawing, or to trace the development of artistic themes over a specific period of time either within one artists oeuvre or across the oeuvres of several artists. In addition, it should have the ability to easily adjust images to appear in the correct size relative to one another, a function necessary for planning the accurate hanging of works of art in a particular exhibition space, recreating historical displays of art, or even producing fantasy exhibitions with works of art that no longer exist or that cannot be moved from their current locations. Combining these advanced functions with basic image editorial capabilities such as cropping and adjusting the color and light saturation allows art historians more easily to make use of lesser quality photographs taken in the field.

In this paper, we focus on the novel tools and techniques we designed to address the specific requirements.

\section{System OVERVIEW}

We built ARIES to meet the requirements gathered during our needs assessment. The ARIES system consists of the following components: a user-friendly interface; the workspace management module that supports uploading images, saving and opening image groups, managing and sharing work in progress; and tools for image exploration, interactive visual comparison, and metadata exploration.

\subsection{User Interface}

Projects carried out by art historians share the common need to view works of art. When these works are unavailable for study, which is usually the case, art historians must substitute visual surrogates in the form of photographs or digital images. Projects can require from very few to a large number of images. The goals also vary. Sometimes, art historians have large collections of images that they need to browse, and by grouping them into observed categories and relationships, they can make new discoveries. In some cases, they just want to check whether two images represent the same work of art or, perhaps, similar works by the same artist or by two different artists. The ARIES interface gives art historians a place to gather these images and to explore them in many different ways, according to the needs of a given project.

The ARIES interface was inspired in the mock-ups developed by graduate students studying user experience. We created several prototypes, performed tests with actual users to evaluate the components of the interface, and iteratively refined the design. The final and functional design is shown in Fig. 2. This interface fulfills some of the basic requirements and it was the first step towards a computational tool for exploring images of works of art.

The interface (Fig. 2) consists of four permanent views that serve different purposes, a toolbar, and keyboard shortcuts that facilitate some operations. The image menu view (Fig. 2(b)) shows a thumbnail for every image that has been uploaded by the user. These are presented in a three-column grid and can be reorganized by dragging the images around within the view. The purpose of this menu is to make a large number of images easily accessible. When dragged, images can also be dropped on the lightbox canvas (Fig.2(d)) or group menu views (Fig. 2(e)).

The lightbox canvas view is where image exploration and interactive visual comparison take place (Subsection 4.3 . Images within this view can be rotated, scaled and translated.

The group menu view organizes groups of images. In this context, a group is a set of images that users put together in order to systemize their findings as needed for a given project. Once a group is created and named in the top menu of the application, it is added to the group menu view. Images from the image menu view can be added to a group by drag and drop. A group is displayed as a stack of thumbnails showing the last five images added to it. The right mouse button, when hovering over a group, enables other operations, such as visualizing every image in the group, throwing all images within the group to the lightbox canvas, removing a group, and annotating a group with metadata.

The metadata view (Fig. 2(c)) displays and allows users to edit information regarding the works of art represented in the images. It includes a set of specific elements such as author, title, year of creation, provenance, and medium to identify and describe the work of art. Once an image is selected in the lightbox canvas, image menu or group menu, the elements within the metadata view are available to receive the appropriate data by the user on the topright corner of the application. Batch annotations of multiple images with the same metadata is also supported.

Along with the different views, ARIES has a toolbar (Fig. 2(a)) for the user to select the tools they wish to use via mouse or keyboard shortcuts. The shortcuts enable a 


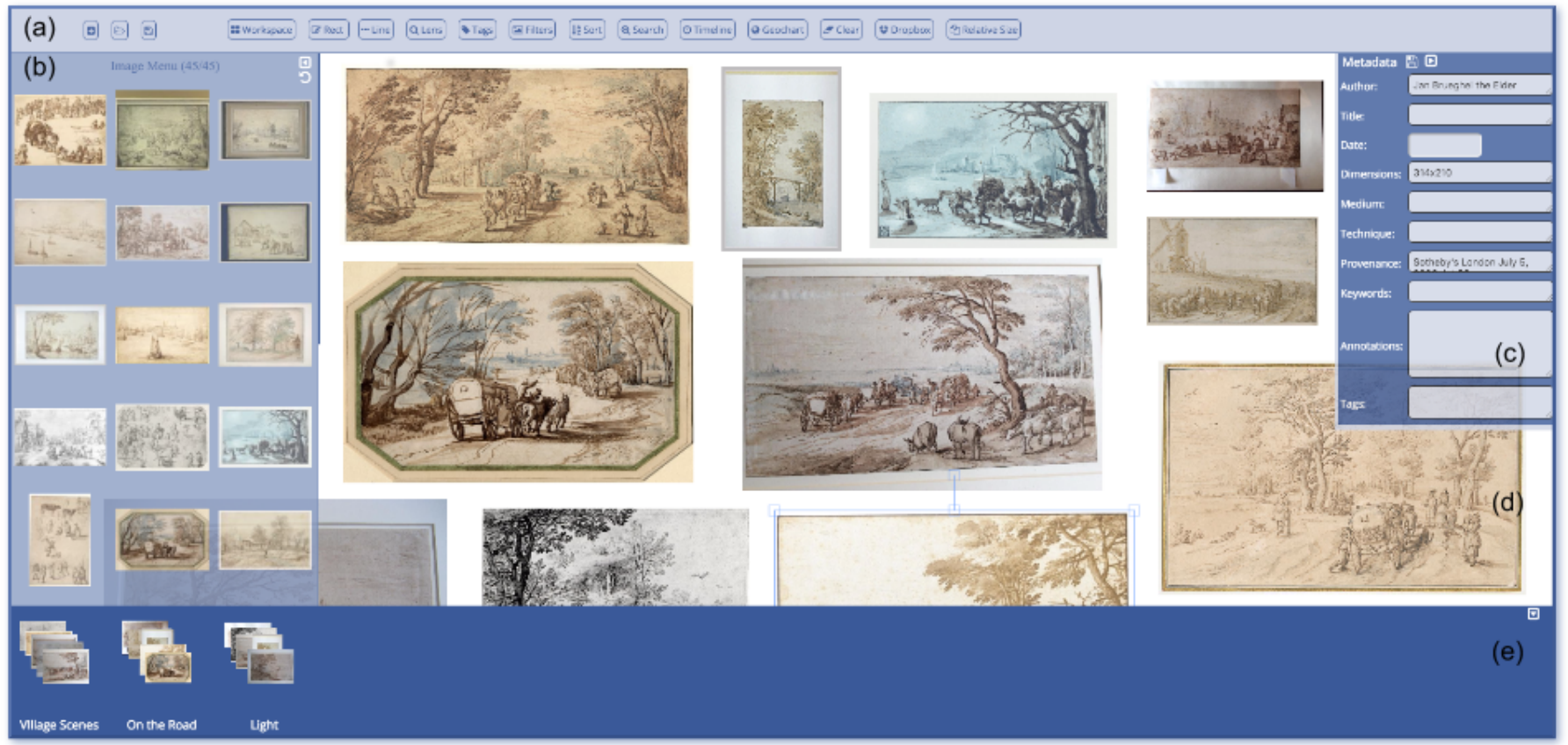

Fig. 2. The ARIES interface includes a toolbar (a) and four views: image menu (b), metadata (c), lightbox canvas (d) and group menu (e). Image menu, metadata and group menu are retractable, enlarging the lightbox canvas. Works of art on the lightbox canvas are displayed in relative size.

user to remove images from all views, enlarge images to fit the entire viewport occluding other images on the lightbox canvas view, and hide/show the three views (image menu, metadata, group menu) to allow for more space to work on the lightbox canvas view.

\subsection{Workspace Management}

The different views in ARIES allow users to organize and compare images. Any image on the user's hard drive can be uploaded, making it available as a thumbnail in the image menu to be explored immediately.

The current state of the application can be saved as a project as soon as the user begins to interact with the system. Saved projects are kept in individual folders and the user has the option of returning the system state to what it was at the moment the project was saved by using the Open Project option. A JSON file is used to store the state of the four views of a project as well as a copies of all images uploaded to ARIES.

Once a project is saved, it is also ready to be shared with other users. To share, the system uploads a saved project to an account on the Dropbox file hosting service. After being prompted to login with a Dropbox account, a directory is created in the user's Dropbox file system and all images present in their project are uploaded to this directory. Images are located at the root along with the JSON file defining the ARIES project. This folder can be shared with other Dropbox users, and the system can download a project folder from Dropbox and load the project back into ARIES.

\subsection{Image Exploration}

An important goal for ARIES is to allow users to organize, compare and annotate art images. Unlike physical light boxes, whose main function is to bring together images for comparison by the human eye, ARIES can leverage digital image processing to manipulate images by size, color, and background. Image comparison consists of two key steps: detecting the similarity or difference among images and interpreting what was detected. In the first step, fine features are automatically or interactively detected. However, interpreting the complex visual details must be driven by a human taking the requirements of the task and context into account. Thus, in ARIES we integrate computing capabilities to detect important details so that the system can guide experts in their analyses.

Interpretation is central to art historians' core task of analyzing works of art images. An art historian analyzing an image is able to make subjective assumptions that would otherwise be difficult to make solely by purelycomputational techniques. For instance, a computational tool can detect that two paintings captured at different occasions by any device (camera or scanner) have differences, but it cannot identify if these differences are due to deterioration of materials used in the original painting over the years or if images are from different paintings. Art experts can use not only their experience, but also their understanding of artistic practice to understand image relationships.

Typical comparative strategies include juxtaposition and superimposition [14]. ARIES implements these strategies to simplify the identification of similarities between different images and interpret complex visual details according a particular task. Images can then be annotated to record new discoveries.

Dynamic overlays: With the dynamic overlay function supported by the lightbox canvas view, images can be placed on top of each other in a single environment. Image opacity is dynamically altered when layered, a function 


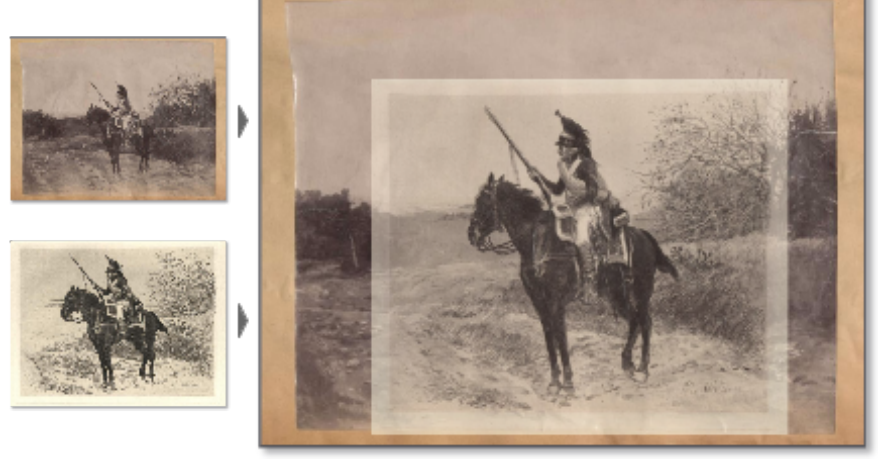

Fig. 3. This image exemplifies the use of the dynamic overlay tool. Two different images were added to the lightbox canvas and one of them was moved on top of the other. The opacity of the top image was modified automatically allowing the user to analyze both images simultaneously. In this example, the alignment was based on the soldier's head.

which is akin to working with physical image transparencies. This function allows the user to see through multiple layers of images, thus recreating an important feature of real light boxes (Fig. 3). Similar shapes that are shown in many images can be manually aligned on top of one another to allow for a better comparison.

The dynamic overlay feature makes an art historian's job much easier when comparing two different visual surrogates for a work of art, i.e., a photograph and a print after a painting, or two different photographs that appear to be of the same painting but may not be. When one is placed on top of the other, the similarities and differences between the print and photograph become immediately apparent. While the dynamic overlay of images is a simple feature, it is an indispensable tool to the art historian's work.

Lens tool: The lens tool reduces the distance between images to be visually compared. By bringing selected areas from two separate images together side by side, this tool simplifies comparisons. Once portions of the images are placed side by side, art historians can address many research concerns including differences in the execution, style, and technique of the works of art, whether they are by the same artist, whether one is a copy of the other, if one or the other shows the work in a conserved state, or if they are two different works of art entirely.

To use this tool, the user selects two images on the lightbox canvas to be displayed in the largest possible sizes, occluding other images. With this new view, the user must manually select two matching points, one in each image, to pinpoint a precise area. These points are the center of two rectangles that highlight the image details of interest to the user. Using the mouse in a swiping motion and keeping the cursor outside of the rectangles, the user can move both rectangles at same time while the system displays a view of the slices of the images contained in the rectangles sideby-side with the rectangle of the reverse image (Fig. 4 . When the mouse cursor is inside the rectangles, the swiping motion alters the sizes of the rectangles to visualize bigger or smaller slices of the original images. The user can also scroll the mouse to zoom in or out the slices. This tool is a special kind of Magic Lens [15] to assist in detecting similarities and differences between images as well as in improving the accuracy of the decision and the eye-saccade time. Fig. 4 shows how an art historian used the tool to compare a digital image of a painting with a digitized photographic reproduction of a similar painting. The tool allowed the art historian to determine that the images reproduced the same painting and as a result she was able to fill in gaps in that painting's provenance.

Rectangle tool: This feature-matching function provides a very simple way for art historians to explore portions of multiple images of the same work of art. By examining different types of images of a work of art, such as $\mathrm{x}$ rays, infra-red reflectograms, prints, drawings and historical photographs, one can make judgements about changes in the artist's intent from drawing to finished work, changes to the work over time, and its representation in other media such as prints.

The user must first manually create a stack of overlapped images and then use the tool to draw one rectangle on the top image (Fig. 5(a) and (b)). After occluding every element on the viewport, the system displays a thumbnail view of the original stacked images and their mapped rectangles in the bottom part of the lightbox canvas view (Fig. 5(c)). On the top, slices of two of the stacked images contained in the rectangles and a heat map relating the slices are shown. The heat map, ranging from white to red, presents the pixel differences between the slices of the two images. The displayed slices can be modified by selecting different images among the thumbnails. With this structure, users can move the relative rectangles of all images in all directions by using the mouse in a swiping motion (Fig. 5(d)) and can zoom into rectangles simultaneously to examine specific details in the set of images. During the replacement, one of the slices displayed on the top of the lightbox canvas stays static while another one is updated with the corresponding portion of the image inside the rectangle, and the heat map is automatically updated. The overview+detail interface [16] allows users to go through all the images according to their previously established mapping, providing a better understanding of the behavior of the image feature detection while retaining the context of the task at hand.

Tags: In order to store discoveries regarding the images, ARIES provides a tag tool. This feature allows art historians to share their observations with other members of a given project or to store them for their own future use. In the lightbox canvas, the user can create a rectangle surrounding a feature in an image and add an annotation related to the feature. A tag, in this context, is the composition of the rectangle and the annotation. The user can create as many tags as desired to record important findings. Once a tag is created, its annotation appears on one of the fields of the metadata view where it can be updated.

Filters: ARIES provides several filters: grayscale, brightness, contrast, color overlay, and edge detection [17]. These filters can be used to emphasize or remove features from images, providing art historians with a better basis for visual comparison.

Note that filters, dynamic overlays, lens and the rectangle tool were designed to meet the specific requirements discussed in Section 3 . 

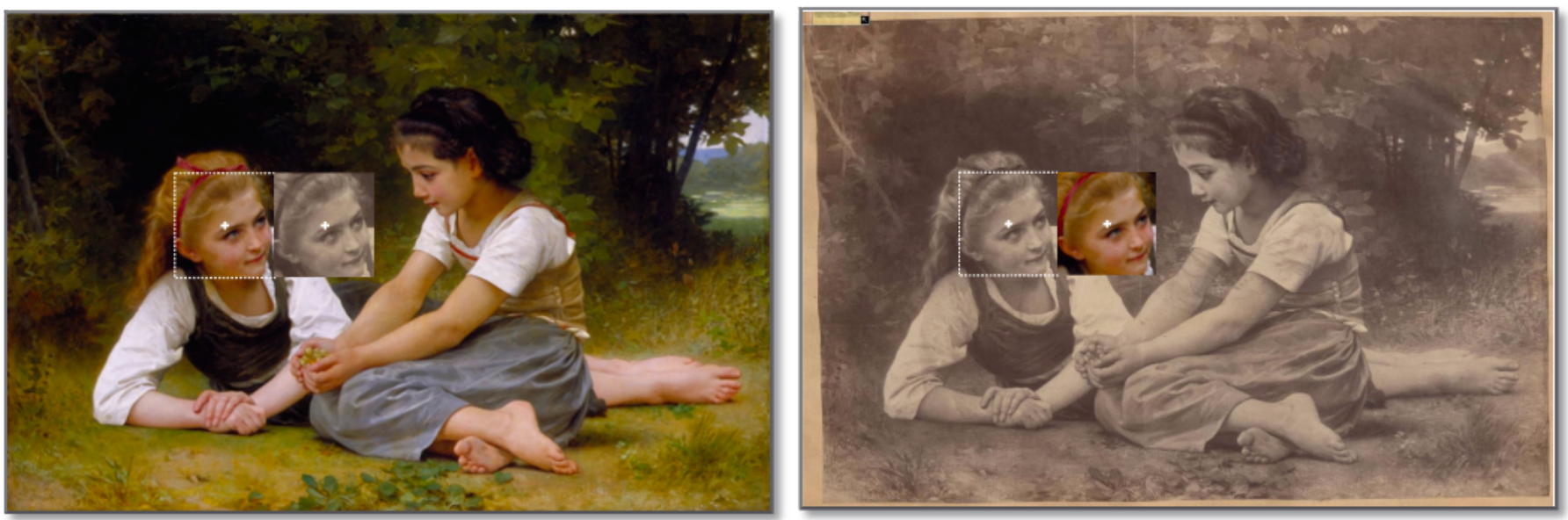

Fig. 4. This figure shows an example of the lens tool applied to two images containing young girls. The matching points are represented by white crosses and centered within the rectangles that surround a precise area for further inspection and analysis. Once the points are marked, the user can move the mouse to update the slices within the rectangles.

(a)
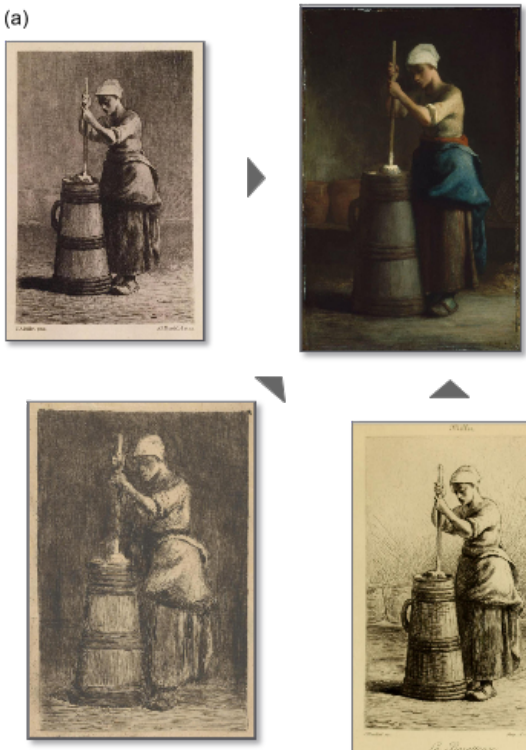

(b)

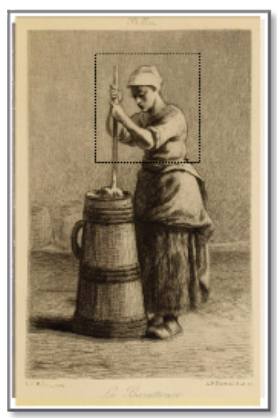

(c)
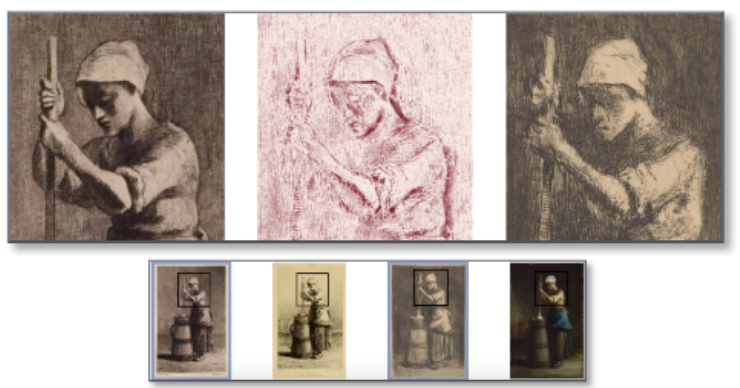

(d)
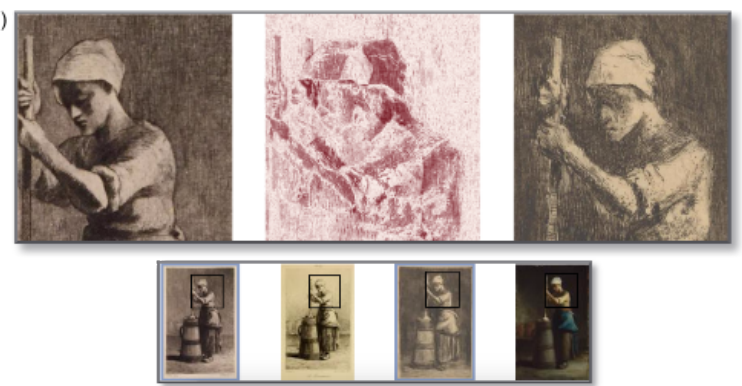

Fig. 5. This figure shows the application of the rectangle tool to the four images in (a). (b) The images are stacked based on the woman's head alignment and one rectangle was drawn around it. (c) shows one of the possible results provided by the tool: in the bottom, the system displays thumbnails of all stacked images and their rectangles, and on the top, slices of original images contained within rectangles are displayed. To help the user identify differences and similarities, a heat map is displayed that represents the pixel differences between the images. (d) shows a different result after moving the rectangles according to the relative position previously established. In this case, only the left image was moved while the right one keeps the same position.

\subsection{Metadata exploration}

Besides exploring the contents of images visually, users can also add to and explore the metadata associated with the images. Below, we describe the metadata exploration functionality supported by ARIES.

Relative Size: This tool allows art historians to see the relative size of one image in comparison with all others on the lightbox canvas. This enables them to understand the relationships between different artworks by answering questions like: Is a given work smaller or larger than another? By how much? This tool is useful for planning the hanging of exhibitions as well as to understand how or where works of art may have been displayed in the past.
Displaying works on the screen as being of the same size can lead to misconceptions. Using the dimensions inserted in the metadata view, the images are rescaled on the lightbox canvas allowing for the real-life proportion of artworks to be evaluated within the application.

Search: This function allows images to be filtered by the different facets present in metadata, including tags. Once the user clicks on search button in the toolbar, the system displays the metadata elements in a dropdown menu and a box where the user must enter the word to search for. The system finds all images stored in the current project that match the search and displays those images in the search menu view that temporarily substitutes the image menu 
view. The drag-and-drop functionality is still available for use with images in search menu view as well as an option to return to previous view.

Sort: The user can sort images from the image menu view or a selected group in the group menu by all categories presented in the metadata view. Once the sort button in the toolbar is clicked, the system displays a drop down menu with the metadata categories and radio buttons, allowing the user to select between the image menu and a selected group. Images are reorganized in their respective view in ascending order of date or alphanumerical order depending on the chosen metadata element.

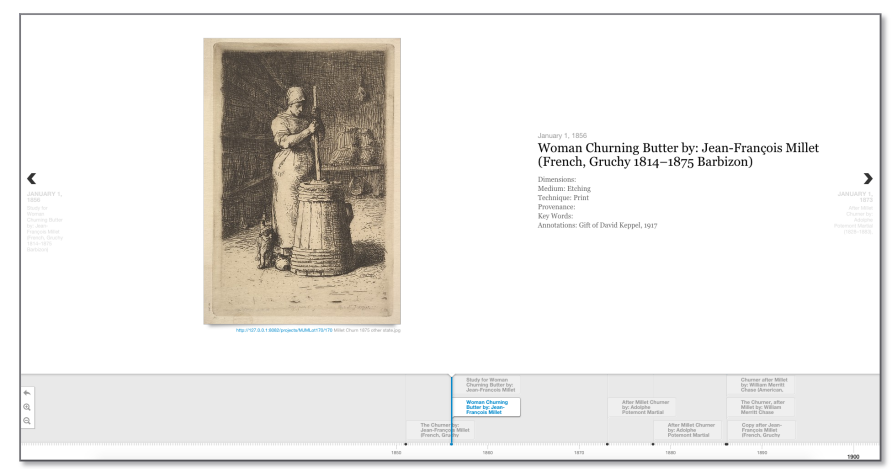

Fig. 6. ARIES's timeline.

Timeline: For art historians, it is important to study the development of an artist's work over time, categorize changes that have occurred to a single work of art over time, and to analyze general trends of artistic techniques and styles over time. Understanding the chronological progression of art and artists throughout the ages helps to determine the "history" of art which can, in turn, shed light on the societies where the art was created.

The timeline tool supports the exploration of artworks over time. ARIES displays a timeline of images from a selected group once the creation dates of the works of art have been entered in the metadata view. By quickly and easily displaying the creation chronology of the given group, stylistic trends or deviations are illuminated. This chronological visualization is designed to display images and their metadata in an interactive way. After selecting a group and clicking on the timeline button in the toolbar, the timeline fits the entire viewport occluding all other views. In the bottom, a chronological line is shown with markers indicating the images on that date. Once the user clicks on the rectangle representing an image (in the timeline), the image and its metadata appear on the top. By clicking on the arrows placed to left or right of the screen, the user can browse all images of the group. Fig. 6 illustrates a timeline created in ARIES by an art historian that shows JeanFrancois Millet's "Woman Churning Butter" and several copies and versions of that work by other artists displayed in chronological order. The timeline quickly enabled new insights concerning the differing thematic interpretations and ways of representing the work of art over time.

Geochart: This function allows for a visualization of the geographical location of works of art over time. It facilitates investigations into the history of artistic styles, the popularity of various artists over time in different countries, and provides insight into the geopolitical history of the exchange of the works of art.

In ARIES, the user can create geocharts in two different ways: with a group of images or with an image. A geochart of one image displays the map of the world with color markers overlapping countries presented in the provenance metadata of the image. Hovering over the markers, the system provides a visualization of markers in more detail. A geochart for a group of images consists of a heat map that represents the number of artworks in each country (Fig. 7). In both cases, a color legend is shown in the bottom part of the canvas. Geocharts are displayed on lightbox canvas, while all other elements are occluded.

The need for search, sort, timeline and geocharts was not identified during the requirements gathering phase. Instead, it was elicited as art historians interacted with the ARIES prototype, and tried to explore and visualize a large number of images.

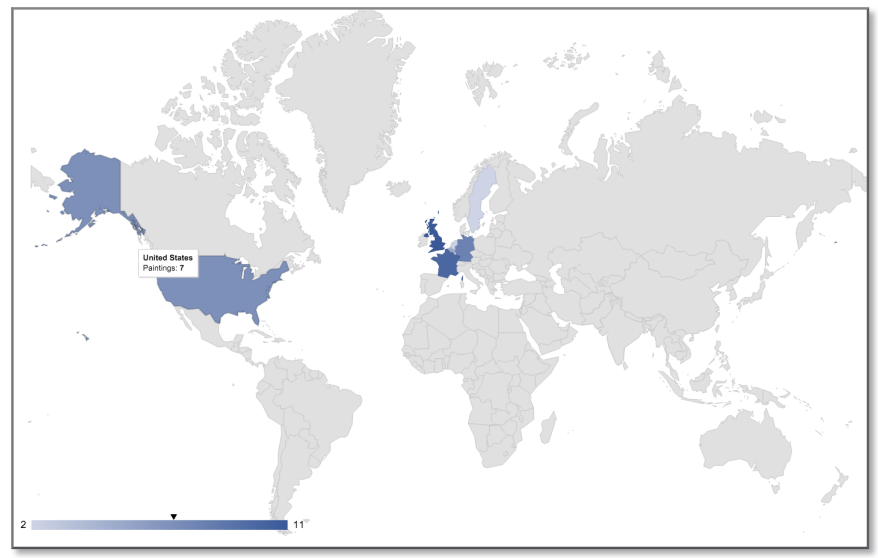

Fig. 7. Geochart of a group created in ARIES.

\subsection{Basic Workflow: Overview}

Tasks carried out in ARIES follow a simple workflow consisting of a series of user actions. First, users create a project and upload the images that they will work with; the images' thumbnails are then shown in the image menu view. Users can drag the images they wish to compare over to the lightbox canvas view. New images can be uploaded and dragged from the image menu at any point. By using the image exploration tools, images inside the lightbox canvas are compared, tags are created to record discoveries, and groups are built to organize the images following user-defined criteria. Metadata is added to images in lightbox canvas, image menu or group menu. The user can browse through the images using their metadata to create timelines and geocharts. The project is then saved and shared. Note that these steps can be rearranged or skipped (it is possible that a user may want to simply compare two images, or organize image groups by visual recognition alone).

\section{IMPLEMENTATION}

ARIES was implemented as a web application. We opted for a web-based application for portability and usability - users need not go through the complexities of installing a system. 
This is especially important for our target users, who have little or no expertise in computing.

The client side was written in JavaScript and HTML5. The jQuery library was used to simplify the JavaScript coding. With the addition of the canvas element with the release of HTML5, JavaScript became a much more viable platform to handle the interactions we needed for the application. The main user interactions are handled through multiple HTML5 canvas elements; the lightbox canvas view uses the Fabric.js canvas library. Fabric provides an image-handling structure that simplified our implementation. Communication with the application server is handled through AJAX, by making GET and POST HTTP requests. The data transferred between the client and the server is encoded in JSON. The dropbox.js library was used to access the Dropbox Core API and establish communications with the Dropbox servers. The use of Dropbox allows ARIES to support seamless sharing among users through a widely-used platform. Other sharing platforms can be easily supported. The timeline and geochart functionality we implemented using Timeline.js and Google GeoChart libraries, respectively.

Since the goal of this work was to design a prototype system to help art historians to visually manipulate and organize art images, we focused our efforts on the client development. To achieve a functional but simple implementation, the server side of ARIES was written in Python and used the framework CherryPy to handle the server deployment and communication with the client. For simplicity, we did not use a database to store the images. Instead, they are maintained in the file system; for each image loaded in a project, ARIES automatically generates and assigns a unique ID that. This information is maintained in a JSON record stored on the server side. Every image request issued by the client is based on the currently available IDs. To achieve interactive performance for the client-server communication, we decided to keep two versions of each loaded image: the original resolution and a thumbnail version with a maximum dimension of 128 pixels. The thumbnail version is used to compose the interface menus while the original ones are used when the user loads an image to the lightbox canvas.

\section{Case Studies}

In this section we present two case studies performed by professional art historians, co-authors of this paper. They demonstrate how ARIES has helped them in their daily workflow.

\subsection{Designing an Exhibition}

The following case study shows how the ARIES system helped curators working in two different countries to collaboratively plan, organize and design an exhibition.

Background: In order to celebrate the 450th anniversary of the birth of the 17th century Flemish artist Jan Brueghel the Elder (1568-1625), one of the most prominent and successful Flemish artists of his time, an exhibition is planned in the city of his birth, Antwerp, Belgium, in 2019. This exhibition will be the first devoted to his drawings. In the past, his works on paper have been presented rather as supplements to his paintings, and yet it was in drawings that he introduced some of his most revolutionary artistic solutions to landscape art, solutions that influenced an entire generation of artists in both the Southern and Northern Netherlands.

The exhibition will also correspond with the launch of an online catalogue of all the artists known drawings. The catalogue and the exhibition are being created jointly by a curator in New York and a curator in Budapest. As a result, many issues arose around the successful sharing of information, ideas, and especially, images. With the help of ARIES, many of these issues have been solved.

As we discussed previously, art historians need to compare, analyze and organize images of works of art. For the Brueghel exhibition, we first needed to decide on a general theme or sequence for the works. Then, we had to gather all the images together in one screen in order to get a sense of what themes the artist was interested in. While currently available tools allow us to do this in a linear fashion, once the images are on one screen, we needed to be able to move them around and create different relevant groupings. With Adobe Bridge, probably the best currently available tool for this type of work, we were able to move images around, but Adobe snapped the images to a grid, making them hard to work with. Further, Bridge did not allow us to separate groupings of images; this had to be done by moving groups of images out of the main screen and into separate folders. This meant that all the images were no longer visible at once on the screen in their new groupings, so that individual folders had to be opened to see them, and the context of the entire oeuvre of the artist was lost.

Once each curator had created their sections of the exhibition, the next step was to share their ideas. Without ARIES, this would have been done by creating a text document, or using Bridge to create $p d f$ files created for each folder. The problem with this process was that the receiving curator had to manually recreate the groups in their environment. Since this back and forth continues for quite some time, the process is quite laborious.

Designing the exhibition with ARIES: With ARIES, collaboration is much easier and more intuitive. Curator A in Budapest uploaded all the images by the artist into the system and, once there, could move them freely around on the lightbox canvas and group them in a thematic fashion (Fig. 2). After saving the project, curator A shared it with curator B in New York. In this way, curator B was able to open the project and see exactly the same screen as A. Rather than having to recreate the subject groups from a typed list or a $p d f$ file, curator B was immediately able to see precisely which images curator A had placed in which subject groups. Curator B in turn opened each grouping individually on the lightbox canvas, and began working with the groupings herself, adding or subtracting works as desired. The back and forth process continued seamlessly until the curators had agreed on the works for each section of the exhibition.

Once the sections were decided, it was necessary to determine the order the works would be hung in. In this case, the curators wanted to hang them in chronological order, in order to follow the way the artist depicted the various themes throughout his lifetime. To assist in this task, 
ARIES created a timeline of the works of art and displayed them in chronological order.

Using Aries, the curators were also able to automatically display the images in relative size to each other on the lightbox canvas (Fig. 2). A relative size display such as this allowed the curators to have a fairly accurate visual idea of what the actual hanging might look like. Once this display was made, again, the two curators were able to save it and send it back and forth to each other for further discussion. The importance of this aspect of ARIES cannot be emphasized enough. Currently there are no tools that do this. In order to get a sense of the sizes of paintings and how they will look next to each other in an exhibition, curators now make print outs of images of the works of art in relative size and hang them on physical mock ups of the exhibition walls.

For designing the exhibition, the metadata view of ARIES was also a very important component. This view let the curators add titles, dimensions, medium, techniques, provenance or location, keywords, and any important annotations regarding the images. This allows curators to share the cataloguing of the works, as well as communicate thoughts and ideas about a particular work with each other through the sharing aspect of ARIES. One last way ARIES was helpful in the creation of the Brueghel exhibition was in the process of asking of loans from other institutions. ARIES helped map the locations of the drawings with its geochart tool (Fig. 7) and a list of possible loans was more easily drawn up from the resulting map.

\subsection{Analyzing Reproductions of Works of Art}

The following case study demonstrates how art historians on our team used ARIES to identify works of art from Mary Jane Morgan's collection, find new information on the history of their ownership (provenance), visualize current locations of the paintings, and make a stylistic analysis of various etched copies by other artists published in promotional materials for Morgan's 1886 auction.

Background: Morgan's collection included contemporary paintings from Europe and the United States with an emphasis on the French Academic School. Academic artists often copied themselves or made close variants of a given composition: altering the size of the work of art, medium employed (pastel, watercolor, oil on panel, etc.), or making slight adjustments in the composition such as changing the direction of a person or an animal depicted. Moreover, many of the paintings were then reproduced as limited edition or unlimited edition prints, and inspired copies by other artists. Trying to identify which version of a work of art was included in Morgan's collection was thus very difficult.

Adding to this confusion, titles of paintings were often translated from French, German, Spanish, Dutch, etc. to English and would even change over time. Further challenges exist because provenance information for the works of art held in both private and public collections are not always complete, measurements not always precise, and attributions change over time. All of these factors made tracing the collection an even more difficult task.

A New York Times article published on March 1, 1886, critiqued the etching reproductions of the paintings in- cluded in the auction catalogue as to whether or not they were close representations of the original works of art. They had judged that some of the etchings captured the spirit of the original paintings more than others. By utilizing the two surviving visual documentations of the paintings from Morgan's sale, the 1886 auction catalogue The Art Collection formed by the late Mrs. Mary J. Morgan, and the art critic Charles de Kay's The Magazine of Art article An American Gallery, published in the same year, we were able to make our own comparisons and judgments. While the representations of the paintings found in the auction catalogue and the magazine were often close in appearance to the original works of art they did not match exactly - they were interpretations by other artists working in different mediums.

Using ARIES to illuminate a private collection: ARIES allowed us to bring together both images of the 1886 etched reproductions of the works of art and their photographic surrogates, and superimpose them for a close analysis. We were able to take modern photographs of paintings we believed had been in Morgan's collection and compare them with etchings made at the time of the auction. In the past we would have performed this task in PowerPoint, where each image has to be adjusted individually. While working in ARIES, this function was performed automatically, thus saving us a considerable amount of time. Further, ARIES provided other tools not available in PowerPoint, such as the relative size, lens, and matching point tools, which helped with other aspects of the comparison. In the past we would have also utilized Pinterest to display images from the collection, however, Pinterest would not have allowed us to reorganize images at will - images are displayed only in fixed groups according to the order they are uploaded. To alter the order of display, we had to create entirely new collections, which was a time-consuming process. With a click of a button, in addition to allowing us to bring together images in one space and annotate them, ARIES allowed for seamless visualizations demonstrating the global redistribution of the paintings which once formed part of Mary Morgan's collection.

Comparing reproductions to images of actual works of art: In the aforementioned 1886 New York Times article, W. H. Shelton's (1840-1932) renderings of Jean L. E. Meissonier's (1815-1891) painting The Vidette, 1812 (lot \#227 in the sales catalog) and J. B. E. Detaille's (1848-1912) A FlagOfficer were reported as favorably representing the paintings. When a photograph of the Meissonier painting and an image of Shelton's etching were analyzed in ARIES, displayed side by side using the lens tool, a different story was revealed. Further when the dynamic overlay was employed, using the horse and vidette as reference points (Fig. 3 ), it was obvious that Shelton had all but forgotten the landscape in his composition. In his rendition, he prioritized the horse and soldier. On the other hand, when Shelton's etching of Detaille's Flag-Officer is compared in ARIES using the lens tool, we observed strong similarities and successful representation of the original work of art. This left us with new art historical questions to ponder, such as why the New York Times felt that Shelton's etching was a faithful 
representation of Meissonier's painting.

In another example we viewed a digital image of a painting from the sale, lot \#170, Jean-Francois Millet's The Churner, beside etchings created by other artists printed in auction catalogues for the purpose of selling the painting. The etchings examined were from sales catalogues dated from 1873, 1879, and 1886. The etching included in the 1886 catalogue was by the American artist, William Merritt Chase (1849-1916), and the etchings included in both the 1873 and 1879 catalogues were by the French artist Adolphe Potemont Martial (1828-1883). We were able to make close observations and track stylistic changes of the works of art by utilizing the lens tool in ARIES - matching corresponding points and then navigating to different corresponding areas of the images simultaneously. The rectangle tool allowed us to a get a closer look at the works of art and facilitated a comparison of the 1886 Chase etching, the Millet painting, and the earlier French etchings (Fig. 5). The heat map demonstrated where the works of art matched up and where they deviated from one another. In ARIES we were able to make a quick observation that the French etching of Millet's painting tended to retain the softness and overall tone of the original work of art, whereas the American rendition appeared edgier, more confined, the churner seemed oppressed, as if working in a dark cellar. After adding the dates of creation and creating a group of images to visualize, the art historian easily created a timeline (Fig. 6). This timeline accentuated the stylistic differences and changes of one theme over time both by the original artist and those creating copies after his work in different mediums.

Discovering missing provenance information: With the help of Aries, we were able to identify and fill gaps in the provenance for William-Adolphe Bouguereau's Nut Gatherers, lot \#154 from Morgan's sale, which now resides at the Detroit Institute of Arts. The provenance listed on the museum's web site does not list ownership before Morgan's 1886 sale. Recently we discovered a photograph of a similar work of art in a 19th century scrapbook of photographic reproductions of paintings that were once offered for sale through Samuel Putnam Avery at his New York gallery at 368 Fifth Avenue. The scrapbook, located in the Rare Books Department at the Metropolitan Museum of Art, has been digitized allowing us to compare a digital image of that painting with one from the painting at the Detroit Museum to see if they represented the same work of art. By utilizing the lens and match point tools in ARIES, we were able to compare the images closely (Fig. 4). In ARIES, the 19th century photograph and the digital image of the painting corresponded to one another perfectly which suggests that Samuel Avery, a person known to have sold pictures to Morgan, once owned the picture, a fact which we were now able to confidently add to the painting's provenance narrative.

\section{Discussion}

System design: The system we designed and implemented combines several interaction mechanisms that were adapted to meet the requirements we gathered from the art historians. For example, the lens tool is an adaptation of magic lens that makes it easier for the users to compare pieces of different images; and the image menu was inspired by Microsoft PowerPoint in making available many images on the left side of the screen easily selectable for analysis and further comparison. An important contribution of this work comes from combining and adapting these mechanisms into a novel unified system that fills an important gap created by the wide adoption of digital images in art research.

Expert feedback: For art historians, a unique benefit of ARIES is that it provides a dedicated out-of-the-box image workspace with an intuitive interface that supports exploration, manipulation, annotation, grouping and sharing of art images in a single environment that can be shared with multiple users. Those to whom it has been demonstrated have wondered why no one had thought of it before, wish it had been available for past projects, and desire a copy immediately. Using existing technology, art historians working with images on computers have had to go through an extremely clunky and laborious process.

Lessons for the community: In moving to a new medium, art historians lost access to a useful 'tool': the physical lightbox. By mimicking the lightbox and adding features that are enabled by digital media (e.g., file organization, image comparison and manipulation), we were able to provide a much improved user experience. Not only has ARIES made easier for art historians' to perform common tasks, but it has also enabled new unexpected uses. The collaborative design of an exhibition across different continents is an example of a novel use art historians discovered, which clearly was not possible with physical lightboxes - as discussed in Section 6 two art historians used ARIES to worked together and interactively on planning and organizing an exhibition.

While ARIES was specifically designed to support art history research, the system is general system and can potentially be useful to organize and compare digital images by professionals (and researchers) in different areas, including designers, photographers, artists, scientists, and digital humanists. For example, one of our collaborators used ARIES to check printers' proofs for an event flyer. Photographers and artists might use the tool to compare, contrast and manipulate images as part of their creative process. Historians comparing scans of multiple versions of both printed texts and incunabula might also find the system useful. In the DARPA Memex program [18], large volumes of images need to be analyzed as experts look for signals of human trafficking in pictures present in online escort ads. Such signals can be found in body features that may indicate an individual is underage, particular markings (e.g., tattoos), or the presence of pictures of different people that have the same background (e.g., this may suggest multiple individuals in the same organization). ARIES can help the experts organize and compare the images, streamlining the analysis as well as the creation of learning classifiers that automatically identify the signals.

As in any interdisciplinary collaboration, we have also faced challenges, including unfamiliarity with each other's work and the need to learn each other's 'language'. Once we were able to overcome some of these barriers and applied a computer science perspective to the problem, together we were able to develop and refine new ideas that led to an effective solution. 


\section{Conclusion}

In this paper we introduced ARIES, an innovative system that helps art historians explore and organize collections of images of artworks. Besides discussing our design decisions and describing the system, together with its implementation, we presented case studies carried out by art historians which demonstrate the effectiveness of ARIES and how it greatly simplifies many of the tasks required for art history research and practice.

While the initial prototype provides a significant step towards our original goal, for a wide deployment, several improvements are needed. For example, currently, the user has to manually input metadata for the images; we plan to support additional mechanisms that allow metadata to be imported from different data sources. To improve the collaboration experience, we would like to support realtime interactions, where multiple users can simultaneously work on a shared project and see each other's modifications. Another direction we would like to explore is the use of ARIES in different domains.

\section{ACKNOWLEDGEMENTS}

We thank Professor Dana Karwas and her students, who used our initial requirements for ARIES in their user experience course, and in the process helped develop the initial user experience mock-ups of the system, which served as foundation for our work. We also thank Lucas Brilhante, Felipe Soares Leite, Fernando Silva, and Autumn Wu who contributed to the implementation at different phases of the project. Maria Beatriz Silva narrated the ARIES video. This work was supported in part by the Brazilian Conselho Nacional de Desenvolvimento Científico e Tecnolgico (CNPq), a Google Faculty Award, an IBM Faculty Award, the MooreSloan Data Science Environment at NYU, AT\&T, DARPA award FA8750-14-2- 023, NSF awards CNS-1229185, CNS1405927, and CCF-1533564. Opinions, findings and conclusions expressed in this material are those of the authors and do not necessarily reflect the views of the NSF or DARPA.

\section{REFERENCES}

[1] B. Fuchs, L. Isaksen, and A. Smith, "The virtual lightbox for museums and archives: a portlet solution for structured data reuse across distributed visual resources," Museums and the Web, 2005.

[2] A. C. Smith, B. Fuchs, and L. Isaksen, "Vlma: A tool for creating, annotating and sharing virtual museum collections," Digital Medievalist, vol. 4, 2008.

[3] Cultural institute. [Online]. Available: https://www.google.com/ culturalinstitute/home

[4] J. Saklofske, "Newradial: Revisualizing the blake archive," paj: The Journal of the Initiative for Digital Humanities, Media, and Culture, vol. 2, no. 1, 2010.

[5] G. Ciocca, P. Olivo, and R. Schettini, "Browsing museum image collections on a multi-touch table," Information systems, vol. 37, no. 2, pp. 169-182, 2012.

[6] U. Hinrichs, H. Schmidt, and S. Carpendale, "Emdialog: Bringing information visualization into the museum," Visualization and Computer Graphics, IEEE Transactions on, vol. 14, no. 6, pp. 11811188, 2008.

[7] E. Hornecker, "i dont understand it either, but it is cool-visitor interactions with a multi-touch table in a museum," in Horizontal interactive human computer systems, 2008. TABLETOP 2008. 3rd IEEE International Workshop on. IEEE, 2008, pp. 113-120.
[8] P. Davis, M. S. Horn, L. Schrementi, F. Block, B. Phillips, E. M. Evans, J. Diamond, and C. Shen, "Going deep: Supporting collaborative exploration of evolution in natural history museums," in Proceedings of 10th International Conference on Computer Supported Collaborative Learning, 2013.

[9] T. Geller, "Interactive tabletop exhibits in museums and galleries," IEEE Computer Graphics and Applications, vol. 26, no. 5, pp. 6-11, 2006.

[10] H. Bay, T. Tuytelaars, and L. Van Gool, "Surf: Speeded up robust features," in Computer vision-ECCV 2006. Springer, 2006, pp. 404417.

[11] D. G. Lowe, "Distinctive image features from scale-invariant keypoints," International journal of computer vision, vol. 60, no. 2, pp. 91-110, 2004.

[12] M.-J. Lobo, E. Pietriga, and C. Appert, “An evaluation of interactive map comparison techniques," in Proceedings of the 33rd annual ACM symposium on User interface software and technology (CHI'15). ACM, 2015, pp. 3573-3582.

[13] M. Elias, J. Elson, D. Fisher, and J. Howell, "Do i live in a flood basin?: Synthesizing ten thousand maps," in Proceedings of the SIGCHI Conference on Human Factors in Computing Systems, ser. CHI '08. New York, NY, USA: ACM, 2008, pp. 255-264. [Online]. Available: http://doi.acm.org/10.1145/1357054.1357100

[14] M. Gleicher, D. Albers, R. Walker, 1. Jusuti, C. D. Hansen, and J. C. Roberts, "Visual comparison for information visualization," Information Visualization, vol. 10, no. 4, pp. 289-309, 2011.

[15] C. Tominski, S. Gladisch, U. Kister, R. Dachselt, and H. Schumann, "A survey on interactive lenses in visualization," EuroVis State-ofthe-Art Reports, pp. 43-62, 2014.

[16] A. Cockburn, A. Karlson, and B. B. Bederson, "A review of overview+ detail, zooming, and focus+ context interfaces," ACM Computing Surveys (CSUR), vol. 41, no. 1, p. 2, 2008.

[17] R. C. Gonzalez and R. E. Woods, Digital Image Processing (3rd Edition). Upper Saddle River, NJ, USA: Prentice-Hall, Inc., 2006.

[18] "Darpa memex program," accessed on February 2, 2017. [Online]. Available: http://www.darpa.mil/program/memex 